\title{
Semana de Arte e Cultura no IFSC Araranguá: experiência em tempos de pandemia
}

\author{
Nayara Nunes Salbego ${ }^{1}$, William Boenavides ${ }^{2}$, Emerson Cardoso ${ }^{3}$, \\ Fabiana Fernandes ${ }^{4}$, Karla Goularte ${ }^{5}$
}

\begin{abstract}
Resumo: O projeto de extensão intitulado Semana de Arte e Cultura, realizado em 2020 por docentes do Instituto Federal de Santa Catarina (IFSC), campus Araranguá, efetuou sua 5a edição, sendo a primeira experiência do evento em formato virtual devido à pandemia. Este relato descreve as atividades didáticas que compuseram os trabalhos apresentados no evento também nomeado Semana de Arte e Cultura. Da mesma forma, apresentam-se os objetivos, métodos e procedimentos elencados para o desenvolvimento dos trabalhos, especialmente enfatizando as atividades que culminaram na $V$ Semana de Arte e Cultura. Por fim, trazem-se as percepções resultantes da realização do projeto como uma experiência positiva em tempos de pandemia. Apesar das adversidades, consideram-se as atividades exitosas, como alto número de acessos, engajamento da comunidade e satisfatório aproveitamento formativo.
\end{abstract}

Palavras-chave: Arte. Cultura. Evento. Extensão. Pandemia.

Área Temática: Cultura.

\section{Week of Art and Culture at IFSC Araranguá: an experience in times of pandemic}

\begin{abstract}
The outreach project entitled Week of Art and Culture was carried out in 2020 by teachers from the Federal Institute of Santa Catarina (IFSC), Ararangua campus. It was the 5th edition of the project, being the first time of the project performed totally online due to the times of pandemic. This practice report describes the pedagogical activities that were part of the event named alike, specially emphasizing the activities that culminated in the 5th Week of Art and Culture. Finally, perceptions about the project were presented as a positive experience in a pandemic time. Besides all the challenges, the activities of the project were successful and had a high number of participations, engagement of the community and satisfactory advantage of social formation.
\end{abstract}

Keywords: Art. Culture. Event. Outreach. Pandemic.

\section{Semana de Arte y Cultura em IFSC Araranguá: experiencia em tiempos de pandemia}

Resumen: El proyecto de extensión Semana de Arte e Cultura, realizado en 2020 por profesores del Instituto Federal de Santa Catarina (IFSC), campus Araranguá, realizó su 5 a edición, siendo la primera experiencia del evento en formato virtual, a consecuencia de la pandemia. Este relato describe las actividades didácticas que componen los trabajos presentados en el evento también llamado Semana de Arte e Cultura. Asimismo, se presentan los objetivos, métodos y procedimientos enumerados para el desarrollo de los trabajos, destacando especialmente

\footnotetext{
${ }^{1}$ Professora de Inglês do Instituto Federal de Santa Catarina, campus Araranguá. E-mail: nayara.salbego@ifsc.edu.br

${ }^{2}$ Professor de Português do Instituto Federal de Santa Catarina, campus Araranguá. E-mail: william.boenavides@ifsc.edu.br

${ }^{3}$ Professor de Arte do Instituto Federal de Santa Catarina, campus Araranguá. E-mail: emerson.cardoso@ifsc.edu.br

${ }^{4}$ Professora de Português do Instituto Federal de Santa Catarina, campus Araranguá. E-mail: fabiana.fernandes@ifsc.edu.b

${ }_{5}^{5}$ Professora de Português do Instituto Federal de Santa Catarina, campus Araranguá. E-mail: karla.grundler@ifsc.edu.br
} 
las actividades que culminaron en la V Semana de Arte e Cultura. Finalmente, las percepciones resultantes de la realización del proyecto se plantean como una experiencia positiva en tiempos de pandemia. A pesar de las adversidades, se consideran actividades exitosas, como un elevado número de accesos, compromiso comunitario y una formación satisfactoria.

Palabras clave: Arte. Cultura. Evento. Extensión. Pandemia.

\section{INTRODUÇÃOO}

A Semana de Arte e Cultura no IFSC Araranguá é um projeto de extensão da área de cultura que se consolidou como um importante evento artístico e cultural desde 2016. Ela ocorre ao longo do ano letivo e se encerra sempre com um evento no final do ano, aberto a toda comunidade araranguaense e região, abordando diversos temas. Em 2020, o tema foi "Vida e sociedade: a pluralidade de valores e olhares". Já em 2019, o tema foi “A diversidade cultural: do clássico ao popular”. Em 2018 e 2017, tivemos respectivamente como temas: "Som e Arte: voz, musicalidade e expressão" e "Marcas de Identidade: o nosso espelho é a investigação do outro". Em 2016, com a primeira edição do evento, o tema foi um dos mais amplos, abordando a arte e a cultura em seus mais diversos aspectos. Com base nesse histórico, foi na última edição, em 2020, uma das mais desafiadoras, realizada em plena pandemia, que os coordenadores e organizadores do evento conseguiram reunir dados relevantes sobre o alcance do projeto. Mesmo na sua primeira versão totalmente online, foi possível realizar uma série de atividades virtuais síncronas e assíncronas que serão relatadas na sequência deste texto.

A V Semana de Arte e Cultura apresentou resultados do projeto de extensão homônimo. Durante o desenvolvimento do projeto, a equipe executora desenvolveu trabalhos de ensino, pesquisa e extensão com o público interno e externo ao IFSC, os quais culminaram na elaboração e execução do evento. Este, por sua vez, transforma a instituição em um espaço de trocas de experiências artísticas e culturais, envolvendo alunos, servidores e comunidade externa - conectados por meio de atividades que refletem criticamente sobre arte e cultura.

\section{OBJETIVOS}

O objetivo geral da Semana de Arte e Cultura é promover experiências artístico-culturais, envolvendo alunos, servidores e comunidade externa. Para isso, dentre os objetivos específicos, estão 1) apresentar atividades artísticas e culturais desenvolvidas pelos alunos ao longo do ano letivo à comunidade externa (curtas, teatro, música, literatura, poema, fotografia, desenho, videoarte, histórias em quadrinhos, pintura, dança, escultura, etc.); 2) introduzir manifestações artísticas externas ao evento, produzidas por pais, familiares e amigos dos alunos, bem como de artistas locais e regionais, como forma de fomentar outras produções artístico-culturais; 3 ) incentivar participação externa nas atividades desenvolvidas no projeto; 4) avaliar, com cunho formativo, os alunos do IFSC com participação ativa no evento; 5) analisar as atividades propostas e executadas pelo projeto, principalmente a V Semana de Arte e Cultura do IFSC Araranguá. 


\section{METODOLOGIA}

Os métodos de execução do evento foram pautados nas proposições básicas dos PCN (Parâmetros Curriculares Nacionais, 2000) e BNCC (Base Nacional Comum Curricular, 2017) para atividades artísticas que possibilitem a avaliação continuada do alunado e a sua respectiva aprendizagem significativa. Como o evento foi realizado online em tempos de pandemia, os recursos necessários foram todos pessoais, ou seja, da equipe executora e dos participantes do projeto. São exemplos: a) conexão de internet; b) recursos audiovisuais como câmeras, headsets, microfones, computadores, celulares etc.

Para a realização do trabalho, pretendeu-se (1) estabelecer as dinâmicas de contato com as escolas da região, em conversas online ou por telefone com gestores e alunado de cada instituição, assim como estabelecer as dinâmicas de contato com os outros polos do IFSC, de forma virtual; (2) contatar museus e outros espaços culturais da região, com convites para apresentação e à apreciação das atividades expostas; (3) utilizar, como impulso das atividades culturais, um tema gerador que anule o "saber neutro" em prol da problematização dos saberes já construídos previamente; (4) discutir/elencar a proposição das atividades artístico-culturais por parte dos professores envolvidos no projeto (todas as atividades foram de cunho interdisciplinar, visto que potencializaram discussões pertinentes a diversas unidades curriculares); (5) envolver atividades paralelas promovidas por servidores e alunos que, de alguma forma, poderiam integrar o eixo temático proposto; (6) agendar datas, horários e plataformas digitais com o respaldo do núcleo gestor da instituição - mais especificamente, com o Diretor de Ensino e Diretor de Comunicação e Relações Externas - tornando-os disponíveis para a realização dos eventos; (7) convidar os colaboradores externos e agendar as oficinas, palestras, lives, roda conversas, mesas-redondas, propostas; (8) gerir, organizar e monitorar o evento junto à equipe executora; e, por fim, (9) avaliar pedagogicamente e qualitativamente as atividades realizadas.

\section{RESULTADOS E DISCUSSÃO}

A Semana de Arte e Cultura é um espaço de produção e de divulgação de conhecimentos artísticos e culturais, norteado por práticas pedagógicas que envolvem a integração de componentes curriculares de Arte, Língua Portuguesa, Literatura, Língua Estrangeira e Projeto Integrador, com o objetivo de contribuir, de forma significativa, com o desenvolvimento dos alunos por meio de práticas inovadoras. Para Ana Mae Barbosa, "A arte na educação afeta a invenção, inovação e difusão de novas ideias e tecnologias, encorajando um meio ambiente institucional inovado e inovador" (2010, p. 2). Nesse sentido, as experiências artísticas e culturais vivenciadas, potencializam percepções, observações e conhecimentos que auxiliam na compreensão não só de conteúdo das referidas áreas do conhecimento, assim como de outras áreas do currículo, além de eles próprios inseridos no mundo da cultura. 
Assim, entendem-se as experiências artísticas e culturais como cumpridoras de um papel formativo único. Tzvetan Todorov (2009), referindo-se à literatura, mostra-nos o seguinte:

Mais densa e eloquente que a vida cotidiana, mas não radicalmente diferente, a literatura amplia o nosso universo, incita-nos a imaginar outras maneiras de concebê-lo e organizá-lo. (...) ela permite que cada um responda melhor à sua vocação de ser humano. (TODOROV, 2009, p. 23-24)

Constitui esse tipo de manifestação artística, portanto, um bem cultural precioso por reforçar nossa identidade enquanto pertencentes a um grupo, a uma coletividade. Alfredo Bosi (1996), ao analisar a raiz etimológica da palavra "cultura" destaca que ela marca não só uma relação com o passado, a que mais comumente a vinculamos, mas também com o futuro:

De cultum, supino de colo, deriva outro particípio: o futuro, cul-turus, o que se vai trabalhar, o que se quer cultivar. (...) Cultura é o conjunto das práticas, das técnicas, dos símbolos e dos valores que se devem transmitir às novas gerações para garantir a reprodução de um estado de coexistência social. A educação é o momento institucional marcado do processo. A terminação -urus, em culturus, enforma a ideia de porvir ou de movimento em sua direção. (...) Cultura supõe uma consciência grupal operosa e operante que desentranha da vida presente os planos para o futuro. (BOSI, 1996, p. 16)

Num ano marcado pela falta de perspectiva advinda da pandemia e de outros fatores desanimadores que se apresentam para a sociedade contemporânea, os laços humanos reforçados pelas experiências culturais, próximas e distantes temporal e geograficamente de nós, podem nos orientar sobre como seguir em frente sem esquecer o passado que nos constrói. Nesse contexto, foi realizada a V Semana de Arte e Cultura do IFSC, campus Araranguá, a primeira que ocorreu de forma totalmente online.

Diante do panorama exposto, realizaram-se atividades online síncronas e assíncronas, cujas dinâmicas agora relatamos. Quanto às atividades síncronas, destacam-se: live de abertura por meio da plataforma StreamYard com transmissão ao vivo pelo Facebook do IFSC campus Araranguá; apresentação de videopoemas do Romantismo e de radionovelas pelo Google Meet e a realização de duas rodas de conversa, uma sobre textos narrativos (crônicas, contos e relatos pessoais) produzidos pelos estudantes dos primeiros anos do Ensino Médio Técnico Integrado com a temática "Pandemia e isolamento social" e outra sobre a "Representação Negra na Literatura Brasileira"․ Já os trabalhos assíncronos foram disponibilizados num blog, cujo link é https:// semanadearteecultura.blogspot.com/. Alguns deles são (1) cartazes digitais que apresentam pessoas, músicas, filmes e lugares que inspiram nossos alunos; (2) histórias em quadrinhos baseadas em releituras de produções artísticas nacionais; (3) murais digitais em Padlets nos quais eles abordam letras das suas músicas favoritas através de imagens, vídeos, áudios; (4) clipes musicais nos quais cada aluno dança uma parte da música na sua casa; (5) exposição virtual de croquis de figurinos de peças de Nelson Rodrigues, resultado do projeto integrador do $2^{\circ}$ ano do Integrado em Vestuário; (6) série de vídeos sobre literatura brasileira dos séculos XX e XXI 
produzida com alguns recursos de acessibilidade para a comunidade surda (alguns vídeos com legenda ou com tradução em libras); (7) série de vídeos sobre autoras mulheres; (8) produção de vídeos e podcasts sobre os contos de Machado de Assis com as turmas dos $3^{\circ} \mathrm{s}$ anos do Integrado em Vestuário e Eletromecânica; (9) Podcasts sobre obras que marcaram a história da arte ocidental, dentre outros trabalhos. O blog, que foi gerenciado pelos alunos bolsistas do projeto, continua disponível e pode ser acessado para visualização do conteúdo, bem como servirá de referência e repositório para futuras edições do evento.

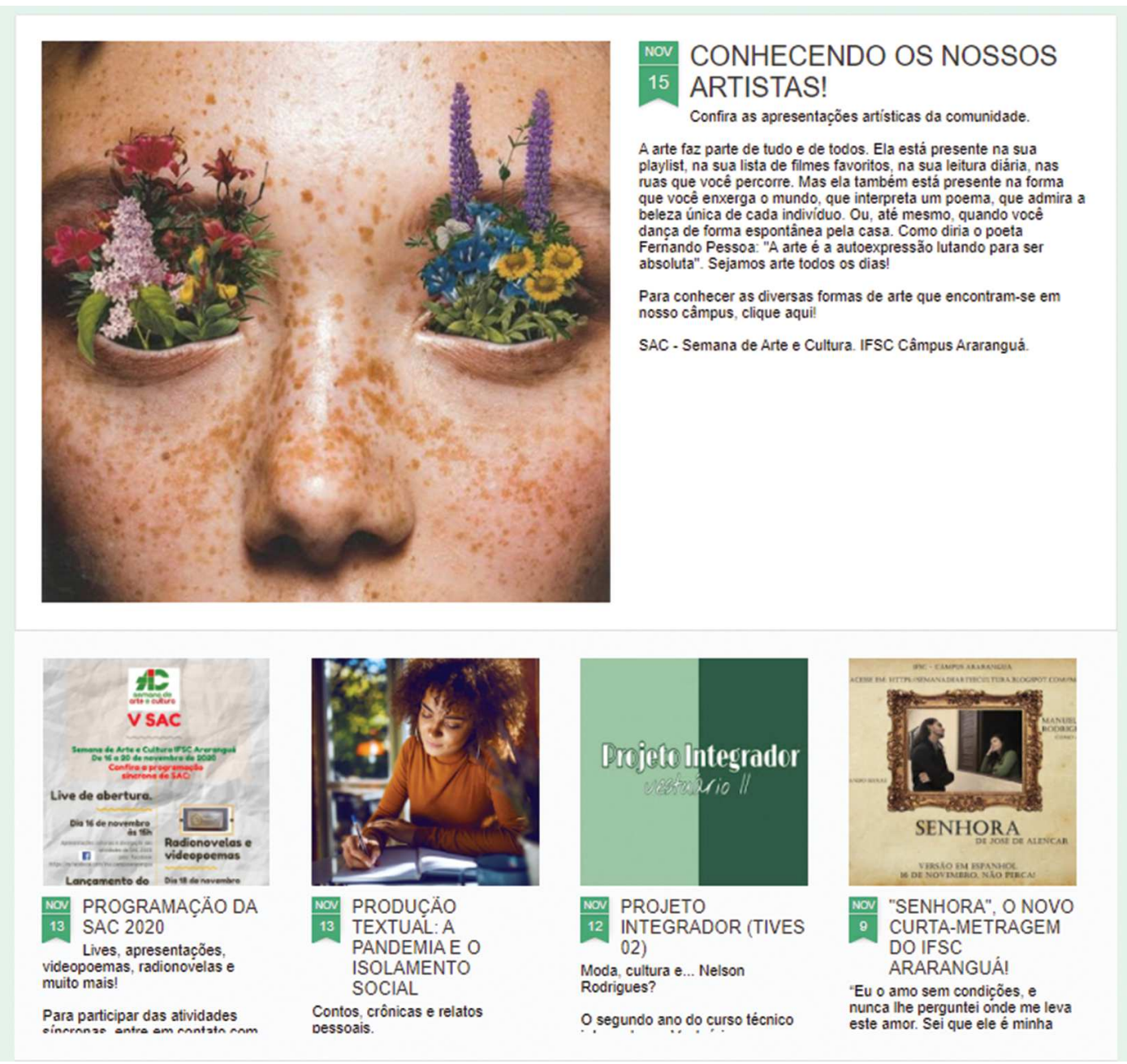

Imagem 1 - Visualização inicial do blog da Semana de Arte e Cultura do IFSC Araranguá. Fonte: autoria própria - printscreen do blog, 2020.

Por fim, o projeto contou com o protagonismo de oito bolsistas, alunos da instituição, cuja atuação foi essencial para o desenvolvimento do evento. Eles tiveram responsabilidades como: auxiliar os professores na disponibilização de suas atividades em plataformas online gratuitas e de acesso aberto; elaborar cartazes e vídeos de divulgação; fazer postagens nas redes sociais do evento; organizar e contatar os artistas da comunidade sobre suas produções a serem apresentadas na live de abertura; enviar mensagens de agradecimento aos artistas que inscreveram trabalhos; interagir com a comunidade por meio de comentários no blog e, durante as atividades 
síncronas, fazer lista de presença, monitorar as entradas de participantes externos, bem como moderar os comentários escritos (com o objetivo de evitar ataques virtuais). Portanto, pode-se afirmar que a atuação dos bolsistas possibilitou que o desenvolvimento do evento acontecesse de forma mais organizada, responsável e produtiva.

\section{CONCLUSÕES}

Como a Semana de Arte e Cultura do IFSC campus Araranguá sempre foi presencial, inicialmente se considerou difícil a participação dos estudantes e da comunidade nas atividades. No entanto, com a criação do blog para o evento, a movimentação nas redes sociais e o gerenciamento dessas plataformas, observou-se uma participação significativa do público.

Os dados obtidos, entre os dias 16 de novembro a 01 de dezembro de 2020, demonstraram que, em cerca de 15 dias, o blog da V SAC 2020 teve 5.191 visualizações. As atividades realizadas pelo Instagram, com um total de 20 publicações, atraíram 384 seguidores. O número de participantes na live de abertura pelo Facebook chegou a 179 curtidas e 183 seguidores. Já a roda de conversa sobre "Pandemia e isolamento social", no dia 17 de novembro, teve 70 participantes. O número de participantes nas apresentações das videonovelas e videopoemas, em forma de evento síncrono no dia 18 de novembro, chegou a 60. O número de participantes na mesa-redonda sobre "Representação Negra na Literatura Brasileira", no dia 20 de novembro, contou com 60 participantes. Com base nos dados coletados, considerou-se que o evento foi um sucesso.

Além disso, o preparo, o estudo e a elaboração dos trabalhos apresentados promoveram motivação nos alunos, pois eles sabiam que suas produções seriam publicizadas. Ademais, o projeto contribui de forma significativa para a comunidade que atinge, sendo que os trabalhos apresentados encorajam reflexão e criticidade sobre arte, cultura, língua e linguagem, temas estes unidos a assuntos atuais, como a questão da pandemia COVID19. Tais preceitos vão ao encontro do que propõe nossa instituição no seu Plano de Desenvolvimento Institucional que apresenta a "Valorização das experiências dos discentes em termos teórico-práticos, metodológicos, tecnológicos, culturais e de cidadania" como um dos princípios da extensão. O referido documento ainda defende que a "extensão é uma dimensão que promove a formação integral do cidadão, uma vez que intensifica a empatia social conduzindo o estudante e o servidor para a realidade econômica e cultural do entorno" (IFSC, 2020-2024, p. $47 ; 56)$.

Com a mudança de evento presencial para o modo de atividade não presencial (ANP), foram feitas diversas adaptações, principalmente no formato das apresentações. Foram criados eventos on-line, lives e o blog da Semana de Arte e Cultura; dessa forma, as exposições e os trabalhos, além de ficarem registrados, puderam ser expostos e apresentados para um maior número de pessoas, sendo visualizados, inclusive, nos EUA e nos Países Baixos. Apesar de a questão da utilização das plataformas oficiais do IFSC para realização de live e postagem nas redes sociais ter sido um desafio, o evento teve muita qualidade e foi proveitoso no sentido amplo 
do termo. Em meio ao caos social causado pelos tempos de pandemia, o evento proporcionou um momento de reflexão sobre o tema do evento "Vida e sociedade: a pluralidade de valores e olhares". Com isso, a V Semana de Arte e Cultura reforçou laços humanos e entrelaçou experiências, permitindo que olhássemos não só para o presente, mas também para o passado em busca de construir uma alternativa mais solidária para o futuro.

\section{AGRADECIMENTOS}

As autoras e os autores gostariam de agradecer à Direção de Extensão e à Pró-Reitoria de Extensão do Instituto Federal de Santa Catarina pelos recursos financeiros que possibilitaram a realização do projeto Semana de Arte e Cultura do IFSC Araranguá.

\section{Notas:}

${ }^{i}$ Ambas as ações foram resultado de uma parceria com o Grupo de Trabalho Temas Transversais do IFSC campus Araranguá.

\section{REFERÊNCIAS}

BARBOSA, Ana Mae Tavares Bastos. A imagem no ensino da arte. 8 ed. São Paulo: Editora Perspectiva, 2010 .

BOSI, Alfredo. Colônia, culto e cultura. In.: Dialética da colonização. 3 ed. São Paulo: Companhia das Letras, 1996.

BRASIL. Base Nacional Comum Curricular. Brasília: MEC, 2017. Disponível em: <http:// basenacionalcomum.mec.gov.br/images/BNC C_20dez_site.pdf>. Acesso em: 22 dez. 2017.

BRASIL, Ministério da Educação. Parâmetros Curriculares Nacionais: Arte. Brasília: MEC/SEF, 2000.

IFSC. Ministério da Educação. Plano de Desenvolvimento Institucional 2020 - 2024.

TODOROV, Tzvetan. A literatura em perigo. Trad. Caio Meira. Rio de Janeiro: Difel, 2009.

Submetido em: 30/03/2021 Aceito em: 11/05/2021. 